\title{
Preoperative computed tomography-guided pulmonary nodule localization augmented by laser angle guide assembly
}

\author{
Stella Chin-Shaw Tsai ${ }^{1}$, Tzu-Chin $\mathbf{W u}^{2}$, Yi-Lin Lai ${ }^{3}$, Frank Cheau-Feng Lin ${ }^{3,4}$ \\ ${ }^{1}$ Department of Otolaryngology, Tungs' Taichung MetroHarbor Hospital, Taichung; ${ }^{2}$ Department of Thoracic Medicine, ${ }^{3}$ Department of Thoracic \\ Surgery, Chung Shan Medical University Hospital, Taichung; ${ }^{4}$ School of Medicine, Chung Shan Medical University, Taichung \\ Contributions: (I) Conception and design: TC Wu, FC Lin; (II) Administrative support: TC Wu, SC Tsai; (III) Provision of study materials or \\ patients: FC Lin; (IV) Collection and assembly of data: FC Lin, YL Lai; (V) Data analysis and interpretation: FC Lin; (VI) Manuscript writing: All \\ authors; (VII) Final approval of manuscript: All authors. \\ Correspondence to: Frank Cheau-Feng Lin, MD, PhD. Department of Thoracic Surgery, Chung Shan Medical University Hospital, 110 Sec.1 Jian- \\ Guo N. Road, Taichung 40201. Email: frnklin@gmail.com.
}

Background: There is an increasing need for thoracic medicine specialists to master preoperative localizations after high rates of sub-centimeter nodules have been positively screened by low-dose CT. The Laser Angle Guide Assembly ${ }^{\circledR}$ (LAGA), an innovative angle reference device for CT-guided pulmonary invasive procedures, has been developed to safely and efficiently aid in the performance of preoperative CTguided localizations (POCTGL).

Methods: The clinical and localization data of patients who received LAGA-assisted POCTGL for pulmonary nodules between May 2015 and June 2018 were collected and analyzed.

Results: One hundred and eighty-seven patients with 266 pulmonary nodules received LAGA-assisted POCTGL. The number of lung nodules localized for one surgery ranged from 1 to 5 , with >1 for $22.1 \%$ of the surgeries. The median nodule size was $6 \mathrm{~mm}$. A hookwire was inserted in $32(12 \%)$ of the nodules. Most (83.1\%) of the localizations were completed with a single puncture. The median angle was 18 degrees. The median and maximum depths of the nodule to pleura were 12 and $60 \mathrm{~mm}$, respectively. The median procedure time was 19 minutes. The successful targeting and field targeting rates were $100 \%$ and $98.1 \%$, respectively. Pneumothorax was noted in 17 (6.4\%) localizations that did not require chest drainage. The multivariable analyses for pneumothorax showed odds ratios of 2.4 (95\% confidence interval, 1.2-4.9) for puncture times/nodule and 10.1 (95\% confidence interval, 2.3-41.7) for tumors adjacent to the fissure, respectively. There was no incidence of hookwire migration.

Conclusions: LAGA enhanced the precision of POCTGL by optimizing targeting precision and decreasing repeated punctures, which minimized complications, such as pneumothorax.

Keywords: CT-guided localization; pulmonary nodules; targeting

Submitted Jun 29, 2019. Accepted for publication Oct 10, 2019.

doi: $10.21037 /$ jtd.2019.10.60

View this article at: http://dx.doi.org/10.21037/jtd.2019.10.60

\section{Introduction}

Lung cancer is the leading cause of cancer deaths internationally and is frequently diagnosed at late stages, which contributes to lung cancer's high mortality rate $(1,2)$. In recent years, low-dose CT screening has been proven to reduce lung cancer mortality and hence gained popularity $(3,4)$. The small, sub-centimeter pulmonary nodules revealed by CT screening frequently require tissue removal for diagnosis and treatment, but these nodules are difficult to detect, either visually or by palpation during thoracoscopy. Therefore, there is a high demand for pre- 
operative localizations that render the nodules 'visible', which many studies have attempted to oblige (5-22).

Several methods have been reported for pre-operative localization, including electromagnetic navigation bronchoscopy (23), ultrasound-guided approaches $(24,25)$, and CT-guided approaches (19). Preoperative CT-guided localizations (POCTGL) that utilize different marker materials have gained popularity in an attempt to make pulmonary nodules 'visible' during invasive procedures since sub-centimeter ground glass nodules are almost exclusively found in CT scans. The diagnostic or treatment procedure usually begins with the selection of a puncture point on the skin surface. Subsequently, a puncture route is created on the selected axial section of the CT scan, according to the planned depth and angle. With an "experienced hand", the puncture is completed (18). To our knowledge, there is no set guideline for the "angle" of puncture. To aid in the precision of punctures, we have developed the Laser Angle Guide Assembly $^{\circledR}$ (LAGA) as a reference for the puncture angle.

Even though thoracic medicine specialists rely on their past experience when performing localizations, most are unfamiliar with proper localization procedures. In this report, we share our experience of utilizing an exact laser guide in place of the "experienced hands" to handle POCTGL procedures.

\section{Methods}

\section{Patients}

Consecutive patients who received POCTGL for small $(\leq 20 \mathrm{~mm})$ pulmonary nodules between May 2015 and June 2018 at a tertiary medical referral center were prospectively included in this study. The electronic chart records and CT scans of localizations on the Picture Archiving and Communication System (PACS) were reviewed. This study was approved by the Institutional Review Board at the Chung Shan Medical University Hospital (No. CS 13221).

\section{Localization procedures}

The details of the procedures were published previously (26). Briefly, patients were fixed well in position with vacuumed bean-bags. All POCTGL procedures were performed under local anesthesia. After the puncture route was planned on the CT scans, the chosen axial level of the body and the target were aligned to the axial laser (red laser, Figure 1A), and the puncture angle was guided with another laser (green laser, Figure $1 A$ ) that was projected from the patient's caudal side and consisted of the planned CT scan angle guided by the LAGA. Then, a 20-G, 90-mm spinal needle (Meditop, Thailand) with an inner solid needle and outer channel was used for the puncture, according to the planned depth controlled by a stopper (Figure 1B). Patent Blue Vital dye (PBV, Guerbet, France, $2.5 \%, 0.2-0.4 \mathrm{~mL}$ ) was used to tattoo the tumor, as well as the visceral pleural surface when the nodule was less than $20 \mathrm{~mm}$ away from the visceral pleura. The inner needle was then withdrawn, the PBV injected with a 1-mL insulin syringe, and the inner needle placed back as soon as possible to prevent the atmospheric air from entering the body (Figure 1C). For those lesions deeper than $20 \mathrm{~mm}$, a hookwire, cut 5-10 $\mathrm{mm}$ longer than the distance from the nodule to the visceral pleura, was implanted along with the PBV at the target nodule (Figure 1D). The hookwire was cut shorter and embedded in the body to prevent it from dropping out. The final CT scan was performed to confirm the accuracy of the localization. The standard localization was achieved when the PBV was in contact with the target nodule on the CT scan, which meant that the tip of the puncture needle should be within $5 \mathrm{~mm}$ of the target nodule.

No oxygen supplementation or bed restriction was needed during the preoperative waiting period after the POCTGL.

\section{Surgery}

All of the surgeries were performed with Video-Assisted Thoracoscopic Surgery (VATS) under general anesthesia. The surgical procedure (either wedge resection, segmentectomy, or lobectomy) was selected according to a consensual principle: when a lesion was smaller than $10 \mathrm{~mm}$ and located at the periphery, wedge resection would be done; segmentectomy would be reserved for a centrallylocated tumor; and for a solid nodule larger than $10 \mathrm{~mm}$, lobectomy would be chosen. Some tiny nodules, such as 3 -mm nodules, were resected because they were removed along with a main suspected lesion in the same surgery.

\section{Data collection}

We collected retrospective data on the following: patients' age; gender; nodule size, location, and number; pathology; localization angle and depth; operation time; and complications. Data on the operation methods and hospital 

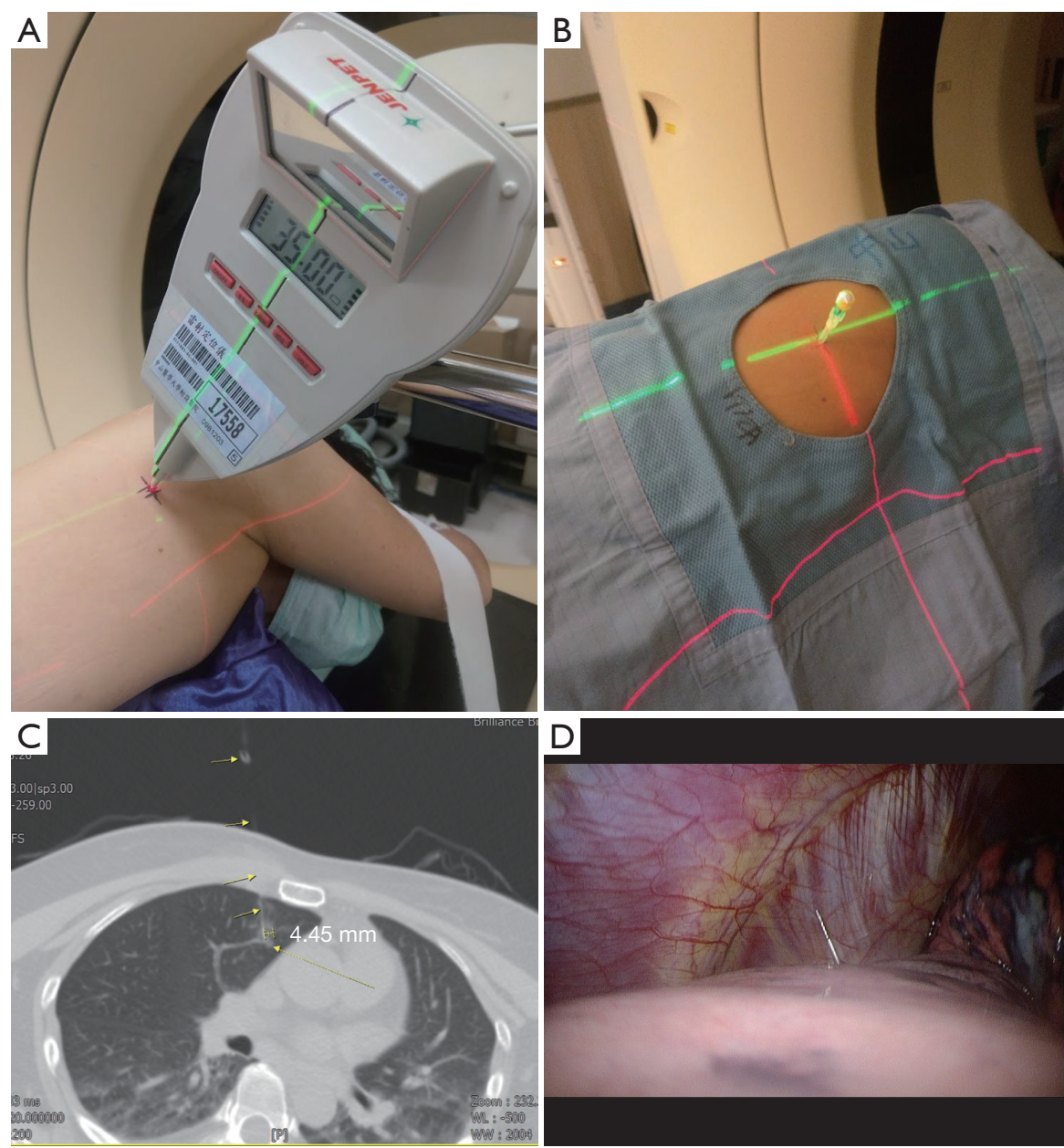

Figure 1 The LAGA-assisted POCTGL. (A) The laser projected to the LAGA according to the angle planned on the CT scan image. (B) The spinal needle inserted into the lung under the guidance of the lasers. (C) The localization of a 5 -mm adenocarcinoma in situ ground glass nodule near the heart. The long arrow from right to left, the nodule. The short arrows from left to right, the partial image of the spinal needle. The PBV was spread $4.45 \mathrm{~mm}$ from the needle. The tip of the needle and the dye contacted the nodule perfectly. The PBV dye was seen from the mediastinal side of the lung during the thoracoscopic surgery. (D) The hookwire cut about 5-10 mm out of the visceral pleural surface. LAGA, laser angle guide assembly; POCTGL, preoperative CT-guided localizations.

length of stay (LOS) were also collected.

During the operation, when the dye was injected from the mediastinum side, diaphragm side, or fissure side of the lung, the locations of the nodules were defined as mediastinum, diaphragm, or fissure, respectively. If the lesion was aimed at through a different lobe, such as through the right lower lobe to target a right upper lobe nodule, the location was defined as cross-lobe.

The time used for localization was defined as beginning from the first tomography and ending at the last checked CT scan.

A successful targeting procedure was defined as completion of the procedure in the CT room with the PBV contacting the lesion, whereas successful surgical field targeting was defined as when the PBV or hookwire were visible in the lung and the target nodules were found in the resected tattooed lung without PBV spillage, fading, or hookwire drop-out $(19,27)$.

Complications were also evaluated. Pneumothorax was defined as any pneumothorax noted in the CT scan localization, even in trace amounts. A challenge was encountered when defining the lung parenchymal hemorrhage because it was difficult to differentiate between the injected dye and blood from the hemorrhage; however, 
Table 1 The results of LAGA assisted POCTGL of pulmonary nodules

\begin{tabular}{|c|c|}
\hline Variables & $\mathrm{N}(\%)$ or median (range) \\
\hline Surgeries & $196(100)$ \\
\hline \multicolumn{2}{|l|}{ Localization numbers } \\
\hline 1 & 141 (71.9) \\
\hline 2 & $45(23.0)$ \\
\hline 3 & $6(3.0)$ \\
\hline 4 & $3(1.5)$ \\
\hline 5 & $1(0.5)$ \\
\hline Total & $266(100)$ \\
\hline \multicolumn{2}{|l|}{ Lobe } \\
\hline RUL & $85(32.0)$ \\
\hline RML & $28(10.5)$ \\
\hline RLL & $58(21.8)$ \\
\hline LUL & $51(19.2)$ \\
\hline LLL & $40(15.0)$ \\
\hline Missing & $4(1.5)$ \\
\hline \multicolumn{2}{|l|}{ Position } \\
\hline Supine & $122(45.9)$ \\
\hline Prone & $96(36.1)$ \\
\hline Left decubitus & $24(9.0)$ \\
\hline Right decubitus & $16(6.0)$ \\
\hline Missing & $8(3.1)$ \\
\hline \multicolumn{2}{|l|}{ Punctures } \\
\hline 1 & $221(83.1)$ \\
\hline 2 & 36 (13.5) \\
\hline 3 & $7(2.6)$ \\
\hline 4 & $2(0.8)$ \\
\hline Mean, SD & $1.2(0.52)$ \\
\hline Hookwire & $32(12.0)$ \\
\hline Cross-lobe & $9(3.4)$ \\
\hline Mediastinum & 35 (13.2) \\
\hline Diaphragm & $34(12.8)$ \\
\hline Fissure & $11(4.1)$ \\
\hline Nodule size (mm) & $6(3-19)$ \\
\hline Angle & $18^{\circ}\left(0-90^{\circ}\right)$ \\
\hline Depth to skin (mm) & $53(22-115)$ \\
\hline Depth to pleura (mm) & $12(0-60)$ \\
\hline Localization time (min) & $19(4-85)$ \\
\hline Localization to surgery (min) & $234(62-507)$ \\
\hline \multicolumn{2}{|l|}{ Radiation dosage } \\
\hline $\mathrm{mGy}^{\star} \mathrm{cm}$ & $470(99-1,622)$ \\
\hline $\mathrm{mSv}$ & $8.5(1.8-29.2)$ \\
\hline
\end{tabular}

LAGA, laser angle guide assembly; POCTGL, preoperative CTguided localizations. from our daily CT-guided biopsy practice, we knew that parenchyma hemorrhage would cause hemoptysis, and therefore hemoptysis was recorded (28). Air embolus was defined as any radiolucent sign that was noted within the vascular system in the CT scan localization.

\section{Statistics}

The statistical analyses were performed with SPSS Statistics for Windows, version 18.0 (SPSS Inc., Chicago, Ill., USA). The categorical data were tested with the $\chi^{2}$ test; for data where the number in a cell was $<5$, Fisher's exact test was used, and for ordinal data, the linear-by-linear association test was adopted. Continuous data were tested with an independent Student's $t$-test. The multiple variable analysis was performed with backward binary logistic regression. The procedure time curve was drawn and tested by linear regression and analysis of variance.

\section{Results}

Between May 2015 and June 2018, 187 consecutive patients who received LAGA-assisted POCTGL were enrolled. Of these, $10(5.9 \%)$ patients had COPD. None of the enrolled patients had active tuberculosis. Most of the patients were female $(136,72.2 \%)$ with an average age of $55.4 \pm 9.4$ years. Data on smoking history were not available. A total of 196 surgeries were performed, including 9 with bilateral lesions. In 303 resected nodules, 266 were localized pre-operatively.

\section{Localization}

The number of nodules localized for one surgery was between 1 and 5 , though $22.1 \%$ of the patients had more than 1 nodule (Table 1). Most of the nodules were located at the right upper lobe (RUL), followed by the left upper lobe (LUL). The proportions of nodules located adjacent to the mediastinum, diaphragm, and fissure were $13.2 \%, 12.8 \%$, and $4.1 \%$, respectively. The cross-lobe approach was chosen for 9 nodules. The median size of the nodules was $6 \mathrm{~mm}$ (mean \pm standard deviation $=6.3 \pm 2.9$ ). The position of localization was mostly supine. A hookwire was inserted in $32(12 \%)$ of the nodules. Most $(83.1 \%)$ of the localizations were completed with a single puncture and $96.6 \%$ with two punctures. The median angle of approach was 18 degrees. The median nodule-to-skin and nodule-topleura depths were 53 and $12 \mathrm{~mm}$, respectively, with maximums of 115 and $60 \mathrm{~mm}$, respectively. The median 


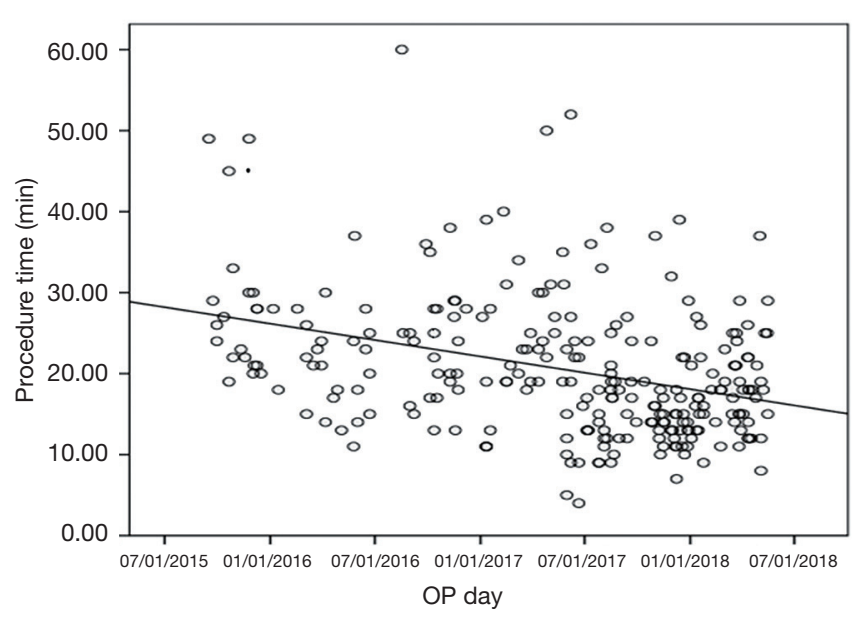

Figure 2 The procedure time of LAGA-assisted POCTGL. Procedure time $\mathrm{t}(\mathrm{min})=27.233-0.011$ (days from 2015/9/16). $\mathrm{R}^{2}$ $=0.124, \mathrm{P}<0.00$. LAGA, laser angle guide assembly; POCTGL, preoperative CT-guided localizations.

Table 2 The complications of LAGA assisted POCTGL

\begin{tabular}{lcc}
\hline Complication & $\mathrm{n}$ & $\%$ \\
\hline Dye spillage (failed localization) & 5 & 1.9 \\
Hookwire migration & 0 & 0 \\
Pneumothorax & 17 & 6.4 \\
Treated pneumothorax ${ }^{*}$ & 3 & 1.1 \\
Hemothorax & 0 & 0 \\
Hemoptysis & 0 & 0 \\
Air emboli & 0 & 0 \\
Vital sign change during waiting & 0 & 0 \\
\hline
\end{tabular}

*, by simple needle aspiration. LAGA, laser angle guide assembly; POCTGL, preoperative CT-guided localizations.

time from dye injection to seeing the dye during surgery was about 4 hours, and after 8.5 hours the PBV could still be visualized clearly. The median time spent on localization was 19 minutes. The localization time significantly decreased, from 27 to 16 minutes, from 2015 to 2018 (Figure 2).

All of the $266(100 \%)$ POCTGL procedures were completed satisfactorily. The successful targeting rate was $98.1 \%$ in the surgical field. There were $5(1.9 \%)$ cases in which the PBV was not recognized within the lung with spillage to the pleura and was re-localized with the aid of the needle puncture holes on both the visceral and parietal pleural surfaces (Table 2). There were no hemothorax, air emboli, or hemoptysis complications among these POCTGL cases for the 266 nodules in 196 surgeries (Table 2). There were no vital sign changes, no need for intubation during the perioperative periods, and no hookwire migration.

Pneumothorax was noted in 17 (6.4\%) localizations and only $3(1.1 \%)$ cases needed temporary treatments with aspiration, whereas the rest were closely followed. The risk factors associated with pneumothorax were analyzed (Table 3). Higher numbers of punctures per nodule, closer proximity of the tumor adjacent to the fissure, and shorter distances from the nodule to the visceral pleura were factors associated with pneumothorax in the univariable analysis. The multivariable analyses showed that the odds ratios for puncture times/nodule and tumors adjacent to the fissure were 2.4 (95\% confidence interval, 1.2-4.9) and 10.1 (95\% confidence interval, 2.3-41.7), respectively. The factors related to pneumothorax were also analyzed based on every single surgery (Table 4). The univariable analyses revealed that the puncture times/surgery and whether the tumor was adjacent to the fissure or mediastinum were contributing factors for pneumothorax. The multivariable analyses disclosed that an odds ratio of 1.8 (95\% confidence interval, 1.2-2.6) for puncture times/surgery and an odds ratio of 8.1 (95\% confidence interval, 1.9-33.3) for any nodule adjacent to the fissure were factors associated with pneumothorax.

\section{Surgery}

There were 196 thoracoscopic surgeries performed on 187 patients (Table 1). No conversion to thoracotomy was needed. There were 274 wedge resections and 21 anatomic resections. Some segmentectomy procedures were also inducted by the POCTGL. Some cases had anatomic resection and wedges in the same surgery. Multiple nodules may have been included in one resection. The median operative time was 105 minutes. The median hospital stay was 5 days. No 30-day mortality was reported.

\section{Patbology}

Of these 187 patients, $168(89.8 \%)$ received surgical procedures that were diagnostic. There were 164 (87.7\%) malignant and 4 benign lesions. Of the 303 pulmonary nodules, $223(73.6 \%)$ were lung cancers (Table 5) and minimally invasive adenocarcinoma $(27.7 \%)$ was the most frequent finding, followed by adenocarcinoma in situ (22.1\%). 
Table 3 Iatrogenic pneumothorax, by nodule

\begin{tabular}{|c|c|c|}
\hline Variables & n (\%) & $\mathrm{P}$ \\
\hline$\chi^{2}$ test & $17 / 266(6.4)$ & \\
\hline Hookwire & & 0.262 \\
\hline 1 & 3/32 (9.4) & \\
\hline 0 & $14 / 271(5.2)$ & \\
\hline Mediastinum & & 0.119 \\
\hline 1 & 4/35 (11.4) & \\
\hline 0 & 13/268 (4.9) & \\
\hline Diaphragm & & 0.409 \\
\hline 1 & $1 / 34(2.9)$ & \\
\hline 0 & 16/269 (5.9) & \\
\hline Fissure & & $0.002^{*}$ \\
\hline 1 & $4 / 11(36.4)$ & \\
\hline 0 & 13/292 (4.5) & \\
\hline Cross lobe & & 0.085 \\
\hline 1 & 2/9 (22.2) & \\
\hline 0 & $15 / 294(5.1)$ & \\
\hline Position & & 0.092 \\
\hline Supine & $5 / 122(4.1)$ & \\
\hline Prone & 8/97 (8.2) & \\
\hline Left decubitus & 2/24 (8.3) & \\
\hline Right decubitus & $1 / 16(6.3)$ & \\
\hline POCTGL doctor & & 0.385 \\
\hline$A$ & $5 / 48(10.4)$ & \\
\hline B & $3 / 30(10)$ & \\
\hline $\mathrm{C}$ & $0 / 17(0)$ & \\
\hline Lobe & & 0.582 \\
\hline RUL & 7/91 (7.7) & \\
\hline $\mathrm{RML}$ & $1 / 30(3.3)$ & \\
\hline RLL & $3 / 64(4.7)$ & \\
\hline LUL & $5 / 56(8.9)$ & \\
\hline LLL & $1 / 42(2.4)$ & \\
\hline Lobe (bilateral combined) & & 0.089 \\
\hline Upper & $12 / 136(8.8)$ & \\
\hline Middle and lower & $5 / 126(4)$ & \\
\hline
\end{tabular}

Table 3 (continued)
Table 3 (continued)

\begin{tabular}{|c|c|c|}
\hline Variables & $\mathrm{n}(\%)$ & $\mathrm{P}$ \\
\hline Puncture times ${ }^{\dagger}$ & & $0.0002^{*}$ \\
\hline 1 & $10 / 215(4.7)$ & \\
\hline 2 & $5 / 36(13.9)$ & \\
\hline 3 & $1 / 7(14.3)$ & \\
\hline 4 & $1 / 2(50.0)$ & \\
\hline \multicolumn{3}{|c|}{ Student's t-test, mean (SD) } \\
\hline Depth to skin & & 0.09 \\
\hline With pneumothorax & $55.6(16.0)$ & \\
\hline Without & $66.1(16.7)$ & \\
\hline Depth to pleura & & $0.03^{*}$ \\
\hline With pneumothorax & $16.1(12.9)$ & \\
\hline Without & $23.2(15.1)$ & \\
\hline Angle & & 0.1 \\
\hline With pneumothorax & $21.9(17.2)$ & \\
\hline Without & $29.1(21.8)$ & \\
\hline \multicolumn{3}{|c|}{ Binary logistic regression, OR (range) } \\
\hline Fissure & $10.1(2.3-41.7)$ & $0.002^{*}$ \\
\hline Puncture times & $2.4(1.2-4.9)$ & $0.014^{*}$ \\
\hline
\end{tabular}

\section{Discussion}

With the increasing number of small pulmonary nodules found via CT screening programs, there is a greater need for pre-operative localizations. Depending on the expertise and availability of experts within the facility, thoracic medicine specialists, surgeons, and pulmonologists may be involved in the POCTGL of pulmonary nodules. LAGA ${ }^{\circledR}$ is an excellent angle reference to assist in performing these procedures. Alternatively, some surgeons have reported successful localizations with costlier navigation systems in a hybrid operating room setting (19).

The performance of this series was compared to those of other published reports (Table 6) (5-18). The present investigation had the largest series with the smallest average nodule size. The procedure was rapid and accurate and the success rate was high with few cases of pneumothorax. Our series demonstrates one of the best POCTGL performances in the literature. 
Table 4 Iatrogenic pneumothorax, by operation

\begin{tabular}{|c|c|c|}
\hline Variables & n (\%) & $\mathrm{P}$ \\
\hline \multicolumn{3}{|l|}{$\chi^{2}$ test } \\
\hline Hookwire & & 0.086 \\
\hline Yes & $5 / 29(17.2)$ & \\
\hline No & 12/166 (7.2) & \\
\hline Mediastinum & & $0.046^{\star}$ \\
\hline Yes & 6/33 (18.2) & \\
\hline No & 11/162 (6.8) & \\
\hline Diaphragm & & 0.811 \\
\hline Yes & $2 / 29(6.9)$ & \\
\hline No & 15/166 (9.0) & \\
\hline Fissure & & $0.009^{*}$ \\
\hline Yes & $4 / 11(36.4)$ & \\
\hline No & $13 / 184(7.1)$ & \\
\hline Cross lobe & & 0.179 \\
\hline Yes & 2/9 (22.2) & \\
\hline No & $15 / 186(8.1)$ & \\
\hline Localize doctor & & 0.393 \\
\hline$A$ & $5 / 36(13.9)$ & \\
\hline B & $3 / 22(13.6)$ & \\
\hline $\mathrm{C}$ & $0 / 12(0)$ & \\
\hline Number of nodules ${ }^{\dagger}$ & & 0.149 \\
\hline 1 & $10 / 139(7.2)$ & \\
\hline 2 & $4 / 46(8.7)$ & \\
\hline 3 & $3 / 6(50.0)$ & \\
\hline 4 & $0 / 3(0)$ & \\
\hline 5 & $0 / 1(0)$ & \\
\hline Puncture times $^{\dagger}$ & & $0.001^{\star}$ \\
\hline 1 & $5 / 113(4.4)$ & \\
\hline 2 & $6 / 54(11.1)$ & \\
\hline 3 & $3 / 14(21.4)$ & \\
\hline 4 & $1 / 6(16.7)$ & \\
\hline 5 & $1 / 2(50)$ & \\
\hline 6 & 1/3 (33.3) & \\
\hline \multicolumn{3}{|c|}{$\begin{array}{l}\text { Binary logistic regression, } \\
\text { OR (range) }\end{array}$} \\
\hline Puncture times & $1.802(1.233-2.632)$ & $0.02^{*}$ \\
\hline Fissure & $0.123(0.030-0.512)$ & $0.04^{*}$ \\
\hline
\end{tabular}

${ }^{*} \mathrm{P}<0.05 .{ }^{\dagger}$ linear by linear association.
Table 5 Diagnosis of lung nodules targeted by POCTGL

\begin{tabular}{lcc}
\hline Pathology & $\mathrm{n}$ & $\%$ \\
\hline Malignancy & 231 & 76.2 \\
Lung cancer & 223 & 73.6 \\
Atypical adenomatous hyperplasia & 18 & 5.9 \\
Adenocarcinoma in situ & 67 & 22.1 \\
Minimally invasive adenocarcinoma & 84 & 27.7 \\
Adenocarcinoma & 54 & 17.8 \\
Metastatic lesions & 8 & 2.6 \\
Benign lesions & 4 & 1.3 \\
Non-disease lesions (non-diagnostic) & 56 & 18.5 \\
Sub-total & 291 & 96 \\
Data missing & 12 & 4 \\
Total & 303 & 100 \\
\hline
\end{tabular}

POCTGL, preoperative CT-guided localizations.

The definitions for successful targeting are still ambiguous (5-18). While most studies have defined successful targeting as the ability to place the markers successfully for surgical reference, the distance from the tumor to the markers remains ill-defined. Yao et al. considered a distance of $15 \mathrm{~mm}$ between the lesion and the localization needle as successful targeting (18). During the surgery, we considered the dye at a distance of $10 \mathrm{~mm}$ away from the nodule as too far and would likely miss the nodule. In contrast with methylene blue, PBV seldom spreads out more than $5 \mathrm{~mm}$ (Figure 1C), even when larger amounts are used (12). Placing the needle adjacent to the nodule was the only way to have the dye in close contact with the nodule; this was our operative definition of successful targeting because it ensured that when the PBV was resected within the safety margin (maximum diameter of the nodule) (29), the nodule would definitely be included. It not only obviated the need and pressure for the surgeon to visually identify the nodule in the resected specimen, but also alleviated the surgeon's doubt regarding whether the nodule was really harvested.

The policy of localization with dye for surface nodules and a hookwire for deeper nodules is well accepted by surgeons (19); however, the high migration rate of the hookwire has been a major concern. The migration of the hookwire might result in missed targets and lead to complications (30). We adopted two safety measures to 


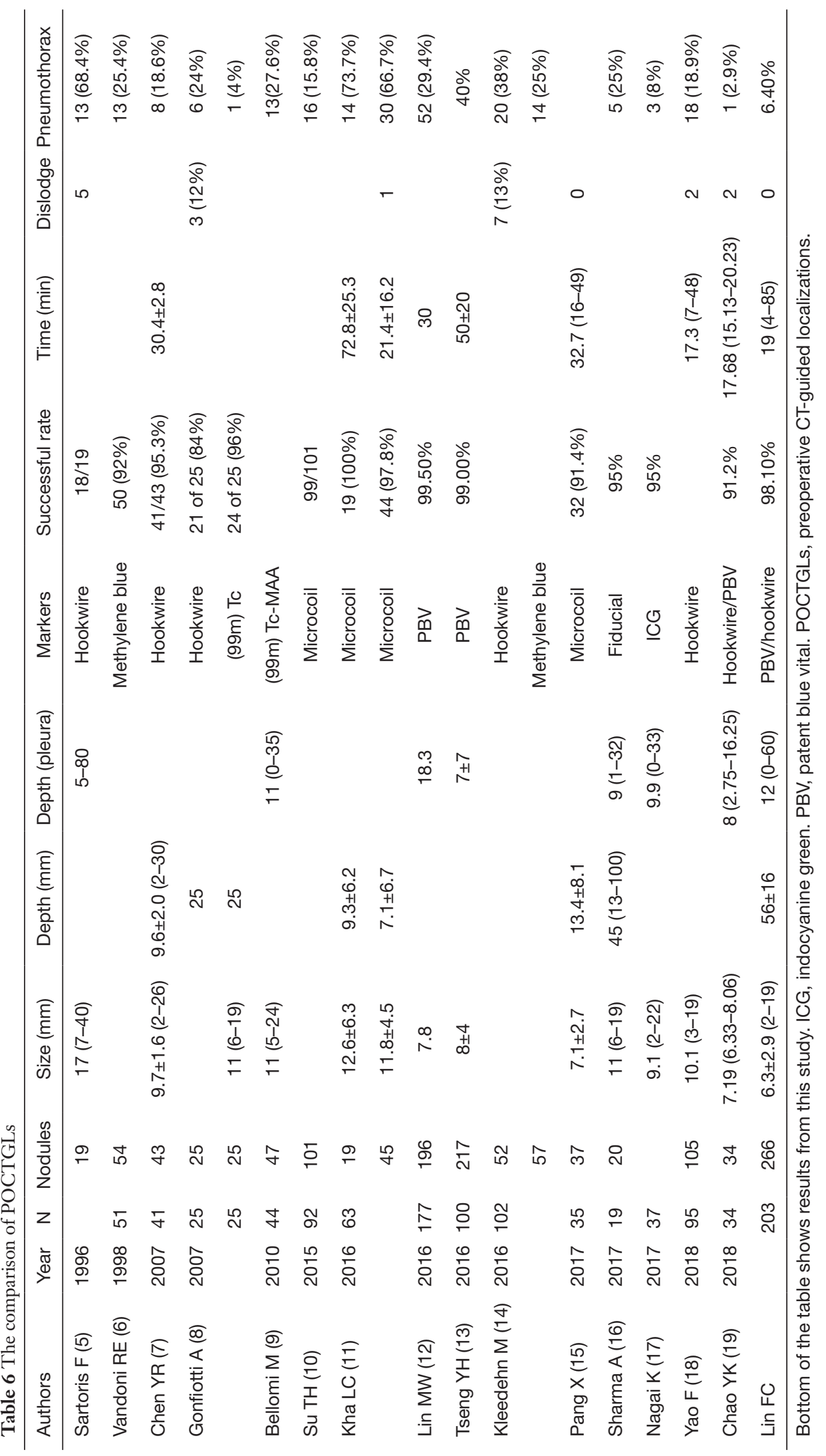


avoid failures of localization. First, we dual-marked the lesion with the PBV and a hookwire. Second, the hookwire was cut shorter and embedded in the body to prevent it from dropping out. As a result, no hookwire migration was observed among our 32 procedures. However, dual markings were only feasible in lesions far from the pleura.

A benefit of using PBV is that it stays in the lung for a long duration without spreading out. Our results suggested that PBV can stay in the lung tissue for more than 8 hours. Lin et al. also reported that PBV stayed in the lung for 561 minutes (12). Despite PBV's long duration, when the needle did not penetrate deep enough into the lung tissue, the dye might still leak out. To prevent this, special attention was paid to ensure that the PBV, in addition to the needle, contacted the lung lesion in the final CT scan. We reviewed the failed cases and found that no dye was detected on the CT scan in these cases. Therefore, we would like to stress the importance of clearly identifying the PBV on the CT scan before the POCTGL is assessed as complete.

In our series, no hemothorax, hemoptysis, or air emboli complications were noted. Air emboli can be fatal and indicate communication between the pulmonary vessels with the atmosphere or airways $(14,30)$. The precautious maneuver of cutting the hookwire shorter and hiding it in the chest wall prevented the atmospheric air from entering the pulmonary vessels. The LAGA further helped the hookwire to reach the planned destination without an accidental interchange between the airway and the pulmonary vessels. Similarly, hemothorax or hemoptysis (lung parenchymal hemorrhage) were avoided by planning the puncture pathway so that the major vessels were circumvented in order to prevent accidental penetrations.

Although pneumothorax could not be completely avoided, we achieved a pneumothorax incidence as low as $6.4 \%$ in our study, which is much lower than that reported in other series (Table 6). According to our statistical analysis, with every additional puncture, we doubled the risk of pneumothorax; this was also observed by Yao et al. (18). Repeated punctures over the adjacent visceral pleura induced pneumothorax. Under the assistance of LAGA, $71.9 \%$ of our POCTGL were achieved in one puncture and $94.9 \%$ were finalized within 2 punctures. Nevertheless, little information is available in the medical literature regarding the association between the number of punctures and complications. Our results showed that performing the POCTGL on nodules adjacent to the fissure had a greater risk of pneumothorax. For nodules adjacent to the fissure, the tip of the needle was inevitably pointed towards the fissure. We speculated that there was a greater chance that the needle would repeatedly touch the same fissure of the visceral pleura during breathing movements. Limited by our study design, these repeated punctures are not shown in our data collection. Unlike localization procedures performed under general anesthesia and positive airway pressure, where pneumothorax tends to happen more frequently (19), our procedures were performed under local anesthesia with negative airway pressure, and meticulously preventing the outside air from entering the chest cavity during the localization procedure was also key in preventing pneumothorax.

In addition to the decreased prevalence of procedurerelated complications, our average procedure time was shorter than that of other studies (Table 6); $(7,11-13,15,17)$. Interestingly, our first-year resident completed 5 LAGAassisted POCTGL procedures, and there was a lack of associated complications, $100 \%$ targeting, and a median procedure time of 19 minutes (data not shown). Therefore, employing LAGA could improve the efficiency of POCTGL independent of the operator's experience.

A distinct advantage of using LAGA was that it replaced the need for real-time fluoroscopy in POCTGL and thus reduced the medical staff's radiation exposure. POCTGL is seldom performed by non-radiologists; however, since the introduction of low-dose CT lung cancer screening, the hybrid operating room and the electromagnetic navigation bronchoscopic system have gained popularity, and so localization may become a skill that thoracic surgeons and pulmonologists have the advantage of mastering $(31,32)$.

A limitation of this study is that it is a one-arm, singlecenter study. A randomized trial that compares LAGA-aided POCTGL against other methods, such as the hybrid operating room and alternative methods of localization, and includes economic considerations may be warranted in the future.

\section{Conclusions}

LAGA enhanced the precision of POCTGL. It reduced the need for repeated penetrations, diminished the chance of pneumothorax and other complications, and increased the rate of targeting success and efficiency.

\section{Acknowledgments}

None. 


\section{Footnote}

Conflicts of Interest: The authors have no conflicts of interest to declare.

Ethical Statement: The authors are accountable for all aspects of the work in ensuring that questions related to the accuracy or integrity of any part of the work are appropriately investigated and resolved. This study was approved by the Institutional Review Board at the Chung Shan Medical University Hospital (No. CS 13221).

\section{References}

1. Barta JA, Powell CA, Wisnivesky JP. Global epidemiology of lung cancer. Ann Glob Health 2019;85:8.

2. Pisani P, Parkin DM, Bray F, et al. Estimates of the worldwide mortality from 25 cancers in 1990. Int J Cancer 1999;83:18-29.

3. Lin FC, Chen CY, Tsai SC. Lung Cancer Screening: Where we are and unanswered questions. SM J Pulm Med 2016;2:1024.

4. Aberle DR, Adams AM, Berg CD, et al. Reduced lungcancer mortality with low-dose computed tomographic screening. New Engl J Med 2011;365:395-409.

5. Sartoris F, Cittadini G, Saitta S, et al. CT-guided needle localization of lung nodules for thoracoscopic resection. Eur Radiol 1996;6:420-4.

6. Vandoni RE, Cuttat JF, Wicky S, et al. CT-guided methylene-blue labelling before thoracoscopic resection of pulmonary nodules. Eur J Cardiothorac Surg 1998;14:265-70.

7. Chen YR, Yeow KM, Lee JY, et al. CT-guided hook wire localization of subpleural lung lesions for video-assisted thoracoscopic surgery (VATS). J Formos Med Assoc 2007;106:911-8.

8. Gonfiotti A, Davini F, Vaggelli L, et al. Thoracoscopic localization techniques for patients with solitary pulmonary nodule: hookwire versus radio-guided surgery. Eur J Cardiothorac Surg 2007;32:843-7.

9. Bellomi M, Veronesi G, Trifiro G, et al. Computed tomography-guided preoperative radiotracer localization of nonpalpable lung nodules. Ann Thorac Surg 2010;90:1759-64.

10. Su TH, Fan YF, Jin L, et al. CT-guided localization of small pulmonary nodules using adjacent microcoil implantation prior to video-assisted thoracoscopic surgical resection. Eur Radiol 2015;25:2627-33.
11. Kha LC, Hanneman K, Donahoe L, et al. Safety and efficacy of modified preoperative lung nodule microcoil localization without pleural marking: a pilot study. J Thorac Imaging 2016;31:15-22.

12. Lin MW, Tseng YH, Lee YF, et al. Computed tomography-guided patent blue vital dye localization of pulmonary nodules in uniportal thoracoscopy. J Thorac Cardiovasc Surg 2016;152:535-44.e2.

13. Tseng YH, Lee YF, Hsieh MS, et al. Preoperative computed tomography-guided dye injection to localize multiple lung nodules for video-assisted thoracoscopic surgery. J Thorac Dis 2016;8:S666-71.

14. Kleedehn M, Kim DH, Lee FT, et al. Preoperative pulmonary nodule localization: a comparison of methylene blue and hookwire techniques. AJR Am J Roentgenol 2016;207:1334-9.

15. Pang X, Xue L, Chen J, et al. A novel hybrid technique for localization of subcentimeter lung nodules. J Thorac Dis 2017;9:1107-12.

16. Sharma A, McDermott S, Mathisen DJ, et al. Preoperative localization of lung nodules with fiducial markers: feasibility and technical considerations. Ann Thorac Surg 2017;103:1114-20.

17. Nagai K, Kuriyama K, Inoue A, et al. Computed tomography-guided preoperative localization of small lung nodules with indocyanine green. Acta Radiol 2018;59:830-5.

18. Yao F, Wang J, Yao J, et al. Reevaluation of the efficacy of preoperative computed tomography-guided hook wire localization: A retrospective analysis. Int J Surg 2018;51:24-30.

19. Chao YK, Pan KT, Wen CT, et al. A comparison of efficacy and safety of preoperative versus intraoperative computed tomography-guided thoracoscopic lung resection. J Thorac Cardiovasc Surg 2018;156:1974-83.e1.

20. Iguchi T, Hiraki T, Gobara H, et al. Transfissural route used for preoperative localization of small pulmonary lesions with a short hook wire and suture system. Cardiovasc Intervent Radiol 2015;38:222-6.

21. Miura H, Yamagami T, Tanaka O, et al. CT findings after lipiodol marking performed before video-assisted thoracoscopic surgery for small pulmonary nodules. Acta Radiol 2016;57:303-10.

22. Imperatori A, Fontana F, Dominioni L, et al. Videoassisted thoracoscopic resection of lung nodules localized with a hydrogel plug. Interact Cardiovasc Thorac Surg 2019;29:137-43.

23. Chen A, Pastis N, Furukawa B, et al. The effect of 
respiratory motion on pulmonary nodule location during electromagnetic navigation bronchoscopy. Chest 2015;147:1275-81.

24. Nakano N, Miyauchi K, Imagawa H, et al. Immediate localization using ultrasound-guided hookwire marking of peripheral lung tumors in the operating room. Interact Cardiovasc Thorac Surg 2004;3:104-6.

25. Kondo R, Yoshida K, Hamanaka K, et al. Intraoperative ultrasonographic localization of pulmonary ground-glass opacities. J Thorac Cardiovasc Surg 2009;138:837-42.

26. Lin FC, Tsai SC, Tu HT, et al. Computed tomographyguided localization with laser angle guide for thoracic procedures. J Thorac Dis 2018;10:3824-8.

27. Park CH, Han K, Hur J, et al. Comparative effectiveness and safety of preoperative lung localization for pulmonary nodules: a systematic review and meta-analysis. Chest 2017;151:316-28.

28. Tomiyama N, Yasuhara Y, Nakajima Y, et al. CTguided needle biopsy of lung lesions: a survey of severe

Cite this article as: Tsai SC, Wu TC, Lai YL, Lin FC. Preoperative computed tomography-guided pulmonary nodule localization augmented by laser angle guide assembly. J Thorac Dis 2019;11(11):4682-4692. doi: 10.21037/jtd.2019.10.60 complication based on 9783 biopsies in Japan. Eur J Radiol 2006;59:60-4.

29. Sawabata N, Ohta M, Matsumura A, et al. Optimal distance of malignant negative margin in excision of nonsmall cell lung cancer: a multicenter prospective study. Ann Thorac Surg 2004;77:415-20.

30. Sakiyama S, Kondo K, Matsuoka H, et al. Fatal air embolism during computed tomography\&\#x2013;guided pulmonary marking with a hook-type marker. J Thorac Cardiovasc Surg 2003;126:1207-9.

31. Bolton WD, Cochran T, Ben-Or S, et al. Electromagnetic navigational bronchoscopy reduces the time required for localization and resection of lung nodules. Innovations (Phila) 2017;12:333-7.

32. Awais O, Reidy MR, Mehta K, et al. Electromagnetic navigation bronchoscopy-guided dye marking for thoracoscopic resection of pulmonary nodules. Ann Thorac Surg 2016;102:223-9. 